Article

\title{
Systematic Understanding of the Mechanism of Salvianolic Acid A via Computational Target Fishing
}

\author{
Shao-Jun Chen * and Ming-Chao Cui \\ Department of Traditional Chinese Medicine, Zhejiang Pharmaceutical College, \\ 888 Yinxian Avenue Eastern Section, Ningbo 315100, China; cuimc@mail.zjpc.net.cn \\ * Correspondence: chenshaojun@hotmail.com; Tel.: +86-574-88223356 \\ Academic Editor: Derek J. McPhee \\ Received: 6 March 2017; Accepted: 12 April 2017; Published: 17 April 2017
}

\begin{abstract}
Salvianolic acid A (SAA) is one of the most abundant water-soluble and potent anti-oxidative compounds isolated from Danshen, a traditional Chinese medicine. A systematic overview of its mechanism of action is yet to be performed. In the present study, the druggability of SAA was measured using the TCMSP server, and potential targets of SAA were identified by PharmMapper and DRAR-CPI. Intersecting targets were then assessed by GeneMANIA and GO pathway analysis, and drug-target-pathway networks were constructed to give a visual view. The results showed that SAA has good druggability, and 13 putative protein targets were identified. Network analysis showed that these targets were associated with cancer, metabolism and other physiological processes. In summary, SAA is predicted to target multiple proteins and pathways to form a network that exerts systematic pharmacological effects.
\end{abstract}

Keywords: Danshen; druggability; network pharmacology; salvianolic acid A; target fishing

\section{Introduction}

Traditional Chinese medicine (TCM) and natural products in general are the best source of active compounds for drug discovery [1,2]. Salvianolic acid A (SAA; Figure 1a) is one such example, and is one of the most abundant water-soluble components extracted from Danshen (red sage, or Radix Salvia miltiorrhiza), a TCM that has been widely used to treat cardiovascular diseases for hundreds of years $[3,4]$. This compound is the most potent anti-oxidative agent among those isolated from Danshen $[5,6]$. Furthermore, SAA is of pharmacological relevance due to its antiplatelet and antithrombosis activities, and it also improves microcirculation. Furthermore, anti-inflammation and antioxidant, myocardial ischemic protection, antithrombotic, neuroprotection and anti-fibrosis activities have been reported, and it can prevent diabetes and associated complications $[4,6]$. For such naturally occurring compounds, knowledge of the toxic or medicinal properties often long predates precise knowledge of targets or mechanisms [1], and there remains much to learn about SAA.

Adoption of computation methodologies for the repositioning of drug molecules in receptors is becoming mainstream because it can save time, money and labour $[7,8]$. Computational target fishing in particular can improve drug discovery and drug design processes and thereby address unmet medical needs [9]. In our previous work, we identified the potential target of capsaicin and tanshinone IIA using computational tools $[10,11]$. As an active compound from TCM, SAA is similarly well suited to this type of analysis.

In the present study, the druggability of SAA was first evaluated using the TCMSP server. Potential targets were predicted by both computational reverse docking and chemical-protein interactome analysis, and overlapping targets identified using both approaches were chosen for further investigation based on gene ontology (GO) and pathway analysis. Finally, we constructed a 
drug-target network to provide a systematic overview of potential targets and mechanisms of action for SAA. An overview of the experimental procedures for SAA target prediction is shown in Figure $1 \mathrm{~b}$.<smiles>[2H][C@@H]1c2cc(O)c(O)c(O)c2OC(=O)[C@@H]1OC(=O)/C=C/c1ccccc1/C=C/c1ccc(O)c(O)c1O</smiles>

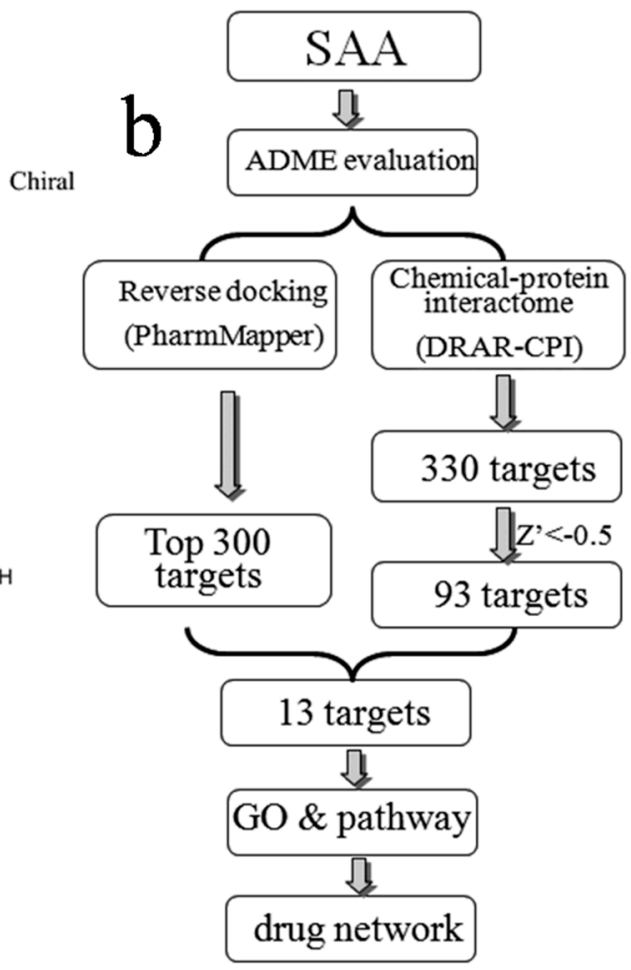

Figure 1. (a) Chemical structure of SAA downloaded from the PubChem database (CID: 5281793); (b) Pipeline for the identification of putative SAA targets that integrates ADME evaluation, reverse docking, chemical-protein interactome, GO, and pathway analyses, and network construction.

\section{Results}

\subsection{ADME-Related Properties of SAA}

TCMSP provides information on important ADME-related properties such as human oral bioavailability (OB), drug-likeness (DL), Caco-2 permeability (Caco-2), blood-brain barrier (BBB) permeability and Lipinski's rule of five (MW, AlogP, TPSA, Hdon, Hacc) [12]. ADME-related properties of ASS were investigated in depth by TCMSP (Table 1). Notably, the DL of SAA was calculated to be 0.7 (Table 1).

Table 1. Pharmacological and molecular properties of SAA.

\begin{tabular}{cccccccccccc}
\hline Name & MW & AlogP & Hdon & Hacc & OB (\%) & Caco-2 & BBB & DL & FSAF & TPSA & RBN \\
\hline SAA & 494.48 & 4.14 & 7 & 10 & 2.96 & -0.56 & -1.62 & 0.7 & 0.4 & 184.98 & 9 \\
\hline
\end{tabular}

\subsection{Identification of Potential Targets}

Potential targets were predicted using two different approaches as described in the methods. Figure $1 \mathrm{~b}$ shows the top 300 potential protein targets of SAA from all 7302 pharmacophore models obtained using PharmMapper, as well as the potential 330 targets identified using DRAR-CPI. In a DRAR-CPI job, a Z'-score threshold of -0.5 indicates favourable targets, and 93 targets met this requirement. Finally, to improve the specificity, a total of 13 potentially interacting proteins identified in both sets of results were selected for further investigation (Table 2). 
Table 2. Putative targets of SAA identified by PharmMapper and DRAR-CPI.

\begin{tabular}{cccc}
\hline Rank & PDB ID & Name & Target Gene \\
\hline 1 & $1 \mathrm{~T} 40$ & Aldose reductase & AKR1B1 \\
2 & 2C3Q & Glutathione S-transferase theta-1 & GSTT1 \\
3 & 2BX8 & Serum albumin & ALB \\
4 & $1 \mathrm{LJR}$ & Glutathione S-transferase theta-2 & GSTT2B \\
5 & 1 XMI & Cystic fibrosis transmembrane conductance regulator & CFTR \\
6 & UEK5 & UDP-glucose 4-epimerase & GALE \\
7 & HE8A & Heat shock 70 kDa protein 1 & HSPA1 \\
8 & 1H0C & Serine-pyruvate aminotransferase & SPAT \\
9 & Glutathione S-transferase P & GSTP1 \\
10 & 11GS & GTPase HRas & HRAS \\
11 & 1A3B & Prothrombin & F2 \\
12 & 1XWK & Glutathione S-transferase Mu 1 & GSTM1 \\
13 & 1R6T & Tryptophanyl-tRNA synthetase, cytoplasmic & WARS \\
\hline
\end{tabular}

\subsection{Analysis by GeneMANIA}

Among the 13 targets and their interacting proteins, it was found that $38.36 \%$ displayed similar co-expression characteristics, and $36.81 \%$ shared the same protein domain. Other results including physical interactions, pathways and co-localisation are also shown in Figure 2.

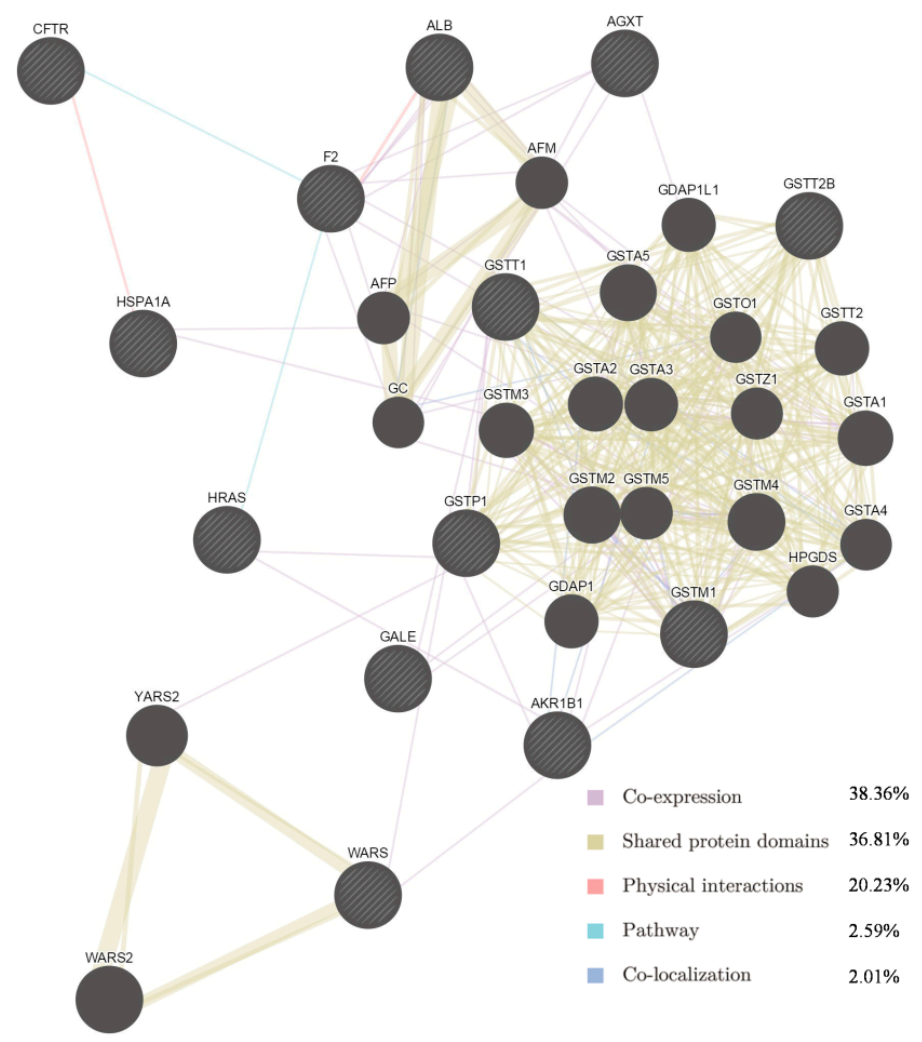

Figure 2. Network of potential SAA targets. Black protein nodes indicate target proteins, and different connecting colours represent different correlations. Functional association of targets was analysed using GeneMANIA. Genes in black circles were submitted as query terms in searches. Grey circles indicate genes associated with query genes.

\subsection{GO and Pathway Analysis, and Network Construction}

To further investigate the 13 identified targets, analysis of interaction network regulation was performed using MAS 3.0. As shown in Figure 3 and Table 3, the top five functions were physiological 
process (GO:0007582), cellular process (GO:0009987), binding (GO:0005488), biological regulation (GO:0065007) and cell (GO:0005623), which together accounted for $45 \%$. As shown in Supporting Table S1, the 13 targets participate in 44 KEGG pathways including glutathione metabolism, metabolism of xenobiotics by cytochrome P450, galactose metabolism, prostate cancer, regulation of actin cytoskeleton and MAPK signalling.

Table 3. GO analysis of potential targets.

\begin{tabular}{ccc}
\hline GO Term & Count & Percentage \\
\hline GO:0007582 Physiological process & 41 & 0.1348684211 \\
GO:0009987 Cellular process & 37 & 0.1217105263 \\
GO:0005488 Binding & 22 & 0.0723684211 \\
GO:0065007 Biological regulation & 20 & 0.0657894737 \\
GO:0005623 Cell & 19 & 0.0625 \\
GO:0044464 Cell part & 19 & 0.0625 \\
GO:0050789 Regulation of biological process & 17 & 0.0559210526 \\
GO:0032501 Multicellular organismal process & 16 & 0.0526315789 \\
GO:0050896 Response to stimulus & 16 & 0.0526315789 \\
GO Other items & 15 & 0.0493421053 \\
GO:0003824 Catalytic activity & 15 & 0.0493421053 \\
GO:0008152 Metabolism & 14 & 0.0460526316 \\
GO:0032502 Developmental process & 11 & 0.0361842105 \\
GO:0051179 Localisation & 10 & 0.0361842105 \\
GO:0043226 Organelle & 9 & 0.0296052632 \\
GO:0051234 Establishment of localisation & 8 & 0.0263157895 \\
GO:0048519 Negative regulation of biological process & 6 & 0.0197368421 \\
GO:0048518 Positive regulation of biological process & 5 & 0.0164473684 \\
GO:0044422 Organelle part & 4 & 0.0131578947 \\
\hline
\end{tabular}

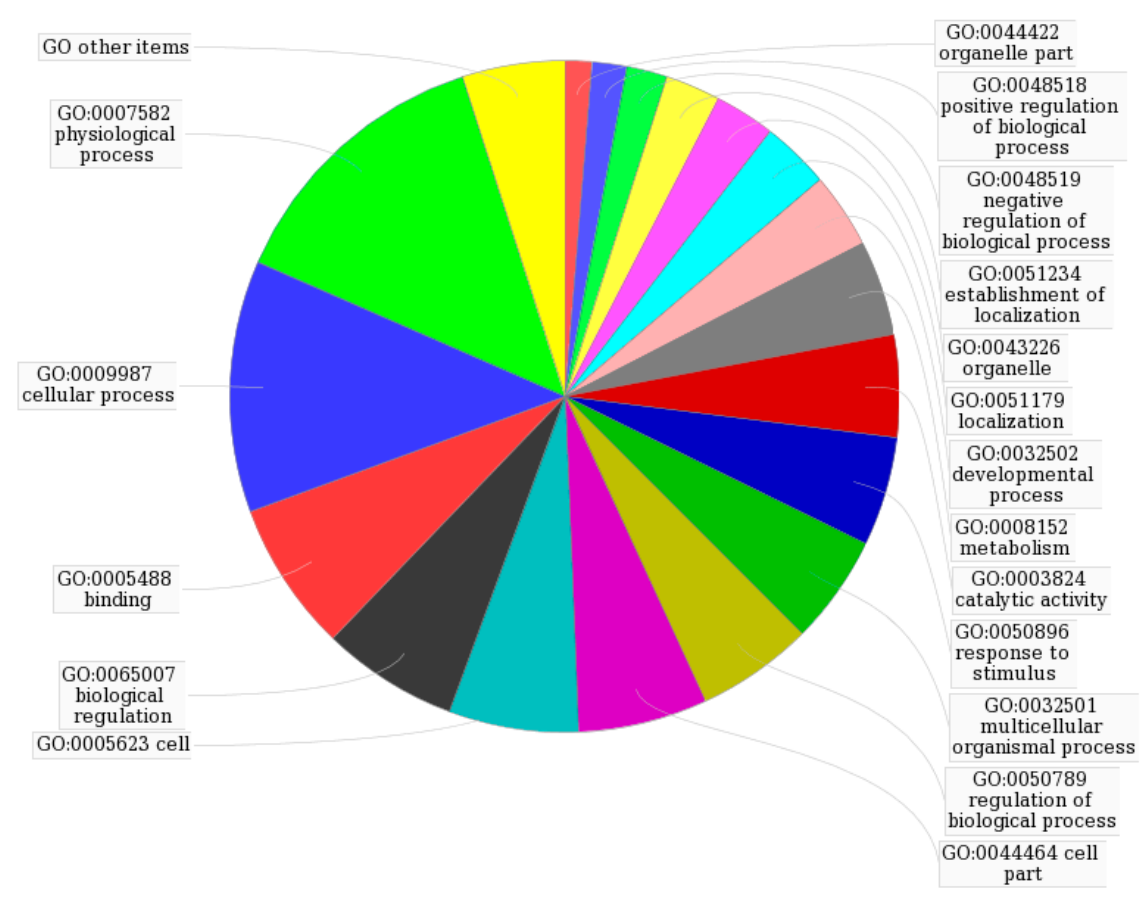

Figure 3. GO map of putative targets following second-level analysis by MAS 3.0.

Based on target fishing and pathway analysis, an entire network was constructed using Cytoscape 3.0. As shown in Figure 4, the interaction network has 59 nodes and 68 edges. The red oblong, green inverted triangles and blue circles correspond to SAA, target proteins and pathways, respectively. 
Drug metabolism - cytochrome P450

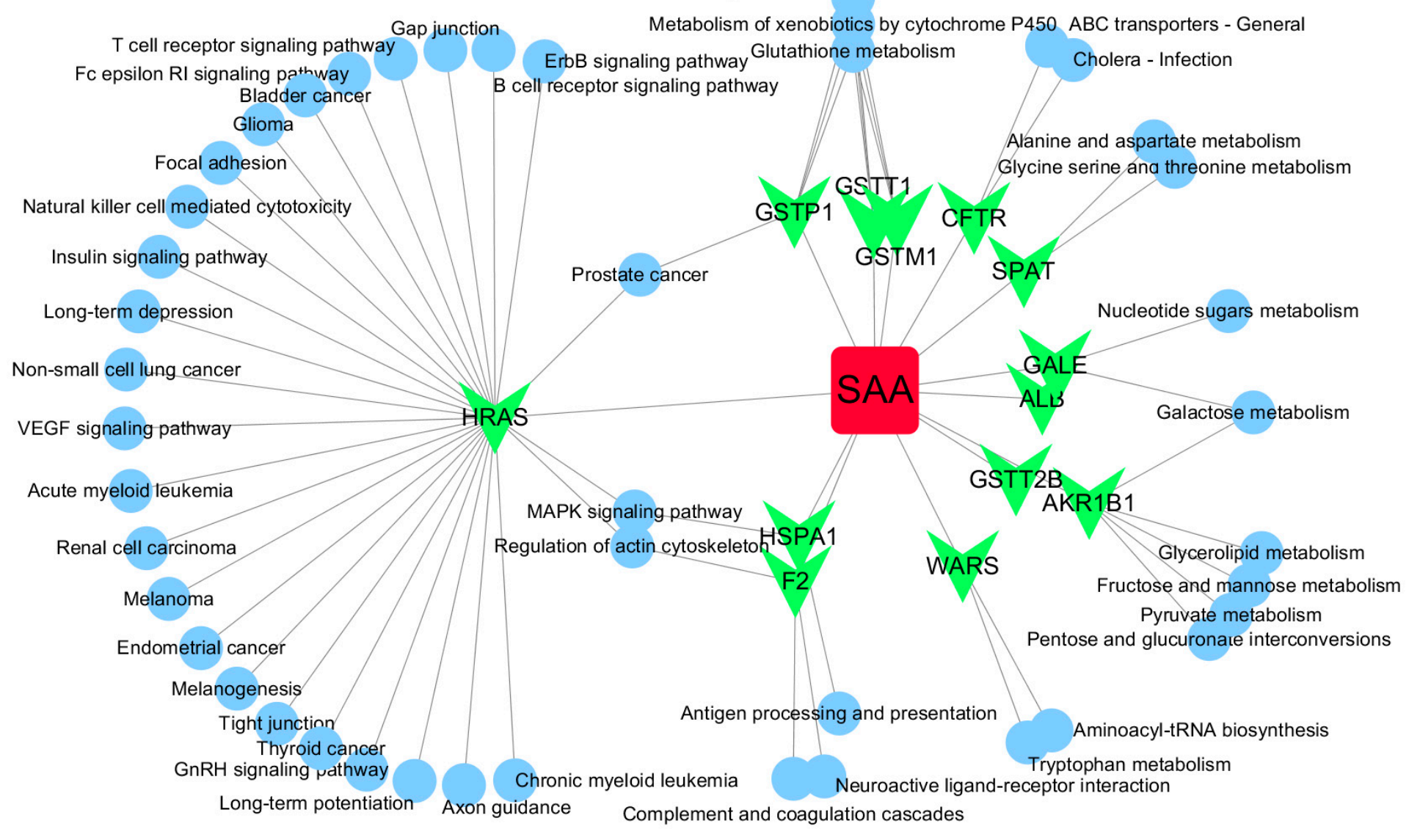

Figure 4. SAA-target-pathway network. Red diamond = SAA, magenta triangle = target proteins, green circles = pathway. 


\section{Discussion}

Poor pharmacokinetics and toxicity are significant causes of costly late-stage failures in drug development, and it is increasingly accepted that these areas should be prioritised in the drug discovery process [13]. In silico approaches can improve our ability to predict and model pharmacokinetic, metabolic and toxicity endpoints, thereby streamlining and accelerating the drug discovery process [13].

Lipinski's "rule of 5" can identify several critical properties that should be considered for compounds with oral delivery in mind $[13,14]$. They are the molecular weight $(\mathrm{MW})<500$ daltons (Da), the calculated $\log P(C \log P)<5$ (or MlogP $>4.15)$, number of hydrogen-bond donors $<5$ and number of hydrogen-bond acceptors $<10[13,14]$. Now, the rule of five is commonly referred to as a "druglike" measure and guideline in drug lead optimisation [13,15]. As listed in Table 1, SAA's properties meet the requirements, which means SAA is a good candidate for drug discovery.

The concept of DL, established from analysis of physiochemical properties or/and structural features of existing small molecule drugs and/or drug candidates, has been widely used to filter out compounds with undesirable properties, especially those with poor ADMET-related profiles [16]. Natural products with inherently good biological properties are understandably receiving increasing attention [16]. Average DL values for Drugbank compounds $\geq 0.18$ have been reported as a criterion for screening bioactive compounds in systems pharmacology-based analyses of TCM [12,17,18]. As shown in Table 1 , the DL value of SAA was calculated to be 0.7 by TCMSP, which is above average, and therefore indicates that SAA may be a promising drug.

Target identification is the first step in drug discovery, and more and more drugs or active compounds are being shown to target multiple proteins $[1,9,19,20]$. Various in silico target fishing methods have been designed and are now widely used for this purpose [21]. As shown in Table 2, 13 putative targets of SAA were screened using computational tools.

Some of the putative SAA targets have been identified previously. For example, aldose reductase (AR) is a major mediator of inflammatory signals induced by oxidative stress, and plays a pivotal role in cellular metabolism, inflammation and cancer [22,23]. In vitro experiments showed that SAA inhibits AR activity and prevents galactose-induced cataract [24]. The GTPase HRas (Table 2) is a Ras isoform that, like other Ras proteins, functions as a GDP-GTP-regulated binary on-off switch that controls cytoplasmic signalling networks affecting a diverse range of cellular processes [25]. S-3-1, a synthetic intermediate of SAA, can suppress the overexpression of the c-myc oncogene, inhibit the function of the Ras oncoprotein, increase the expression of the P53 tumour suppressor, and interrupt P46-associated and mitogen-activated pathways distinct from farnesylation of Ras [26]. Glutathione S-transferases (GSTs) are a family of detoxification enzymes that catalyse the conjugation of glutathione to a wide variety of xenobiotics [27]. Danshensu, another active compound from Danshen, reduces MDA and restores GSH levels and total SH content in the cultured rat lens [28]. A combination of extracts from Danshen act pharmacologically via GST $[29,30]$. As for other putative targets, salvianolate, a highly concentrated form of salvianolic acid B, can inhibit neuronal apoptosis by increasing heat shock protein 22 in a reperfusion-ischemia model [31]. Therefore, the identification of 13 potential protein targets is consistent with multiple targets for SAA. The GeneMANIA results (Figure 3) provided information on co-expression and shared protein domains, and suggested that the targets and their interacting proteins may have identical or similar functions, in agreement with the results of the first step.

There have similar reports about predictive targets of SAA by computational tools. For example, Li developed an approach by combining network efficiency analysis with scoring function from molecular docking to estimate the anticoagulant activities of compounds which includes SAA [32]. A recently published paper also reported that SAA exhibits 8 hepatoprotective targets (BCL2, AKT1, CCND1, SPZ1, COL1A1, CDKN1A, HERC5 and MMP2) connections by in silico-based network pharmacology approach [33]. Notably, AKT1 and MMP3 are also the PharmMapper's results of SAA in our study (Supporting Table S2).

GO and pathway analysis was also conducted using MAS 3.0, and a network was constructed using Cytoscape. As shown in Figure 4, the network also indicated that SAA has multiple targets and 
further implies that it possesses multiple pharmacological activities. Interestingly, HRAS was identified as a potential hub protein in the network (Figure 4). SAA reverses paclitaxel resistance in human breast cancer bytargeting the expression of transgelin2 and attenuating the PI3K/Akt pathway [34], inactivates transgelin2 [35], protects human neuroblastoma SH-SY5Y cells against MPP+-induced cytotoxicity [36] and inhibits the growth of A549 lung cancer cells [37]. These findings indicate that the anticancer activity of SAA likely involves Ras proteins.

\section{Materials and Methods}

\subsection{Evaluation of Drug-Likeness Using the TCMSP Server}

The TCMSP server (http://ibts.hkbu.edu.hk/LSP/tcmsp.php) is a systems-level pharmacology database for TCM that can also calculate ADME-related properties for interesting, naturally occurring compounds [12]. It provides an in silico ADME-systems evaluation model created by Wang's research team, which integrated drug-likeness (DL), oral bioavailability $(\mathrm{OB})$, and Caco-2 permeability and so on $[12,17,18]$.

Drug-likeness (DL) is a qualitative concept used in drug design for an estimate on how "drug-like" a prospective compound is, which helps to optimize pharmacokinetic and pharmaceutical properties, such as solubility and chemical stability [17]. A database-dependent model is constructed based on the molecular descriptors and Tanimoto coefficient (as displayed below) [17,18]:

$$
\mathrm{T}(\mathrm{A}, \mathrm{B})=\frac{\mathrm{A} \times \mathrm{B}}{\|\mathrm{A}\|^{2}+\|\mathrm{B}\|^{2}-\mathrm{A} \times \mathrm{B}}
$$

where $\mathrm{A}$ is the molecular descriptor of herbal ingredients, and B shows the average molecular properties of all molecules in Drug-Bank database [17,18].

Oral bioavailability $(\mathrm{OB})$ is one of the most vital pharmacokinetic properties of orally administered drugs because it plays an important role for the efficiency of the drug delivery to the systemic circulation. Its value was calculated by an in-house model OBioavail1.1 in the TCMSP database [12,17].

For orally administered drugs, one of the greatest problems is movement across the intestinal epithelial barrier, which determines the rate and extent of human absorption and ultimately affects its bioavailability $[12,17]$. The Caco-2 permeability prediction model preCaco2 was applied in the TCMSP database [12,17].

In this study, the chemical name "salvianolic acid A" was entered as the search term and its druggability was analysed at the molecular level.

\subsection{Computational Target Fishing by PharmMapper and DRAR-CPI}

PharmMapper, a reverse docking server, can identify potential protein targets for small molecule compounds via a pharmacophore mapping approach [38]. For a given compound, it can provide the top 300 targets, sorted by fit score in descending order [38]. Meanwhile, the DRAR-CPI server can identify targetable proteins for small molecules via chemical-protein interactome analysis [39]. Both are powerful tools for computational target fishing.

An sdf file for SAA (PubChem CID: 5281793) was downloaded from the PubChem database and separately uploaded to the PharmMapper and DRAR-CPI servers. All parameters were set to default values, and overlapping protein targets identified using both servers were chosen for further investigation.

\subsection{Analysis by GeneMANIA}

GeneMANIA is a flexible, user-friendly web interface for generating hypotheses about gene function, analysing gene lists and prioritising genes for functional assays [40]. After selecting Homo sapiens from the nine optional organisms, the gene of interest in the previous step was entered into the search bar and the results were collated. 


\subsection{GO and Pathway Analysis, and Network Construction}

The Molecule Annotation System 3.0 (MAS 3.0), a web-based software toolkit, can be used to help understand relationships within gene expression data and provide systematic and visual information on the gene of interest. Potential targets were uploaded to the MAS 3.0 server (http: //bioinfo.capitalbio.com/mas3/) following the online instructions, and GO and KEGG pathway information for SAA was generated and collected. For a deeper understanding of the complex relationships among compounds, targets and diseases, networks were constructed and analysed using Cytoscape 3.0.

\section{Conclusions}

In the present study, the DL of SAA was evaluated by TCMSP, and potential targets identified by both PharmMapper and DRAR-CPI were chosen for further investigation. The results showed that SAA may be a good drug candidate, and 13 potential interacting partners were identified that are associated with various pharmacological activities. In addition, GO and pathway analysis was performed and a drug-target association network was constructed. These results indicated that SAA has multiple functions, including anticancer and metabolic activity. Although further studies are necessary to determine the precise interactions, this study provides a systematic and visual overview of possible SAA molecular mechanisms and signaling pathways.

Supplementary Materials: The following are available online. Table S1: KEGG pathways of putative targets identified by MAS 3.0, Table S2: The top 300 potential targets of SAA predicted by PharmMapper.

Acknowledgments: This work was supported by Zhejiang Provincial Natural Science Foundation of China (LY15H280009), Ningbo Municipal Natural Science Foundation (2015A610280) and Zhejiang Pharmaceutical College (ZPCSR2015018).

Author Contributions: Shao-jun Chen conceived and designed the experiments; Shao-jun Chen and Ming-Chao Cui performed the experiments; Ming-Chao Cui analyzed the data; Shao-jun Chen wrote the paper.

Conflicts of Interest: The authors declare no conflicts of interest in association with this manuscript.

\section{References}

1. Schenone, M.; Dancik, V.; Wagner, B.K.; Clemons, P.A. Target identification and mechanism of action in chemical biology and drug discovery. Nat. Chem. Biol. 2013, 9, 232-240. [CrossRef] [PubMed]

2. Shen, B. A new golden age of natural products drug discovery. Cell 2015, 163, 1297-1300. [CrossRef] [PubMed]

3. Ho, J.H.; Hong, C.Y. Salvianolic acids: Small compounds with multiple mechanisms for cardiovascular protection. J. Biomed. Sci. 2011, 18, 30. [CrossRef] [PubMed]

4. Zhang, L.; Zhang, W.; Zhao, Y.; Yang, X.; Fang, L.; Wang, S.; Shi, L.; Yu, X.; Yang, H.; Sun, J.; et al. Research progress of salvianolic acid A. Zhongguo Zhong Yao Za Zhi 2011, 36, 2603-2609. [PubMed]

5. Liu, G.T.; Zhang, T.M.; Wang, B.E.; Wang, Y.W. Protective action of seven natural phenolic compounds against peroxidative damage to biomembranes. Biochem. Pharmacol. 1992, 43, 147-152. [CrossRef]

6. Fan, H.Y.; Yang, M.Y.; Qi, D.; Zhang, Z.K.; Zhu, L.; Shang-Guan, X.X.; Liu, K.; Xu, H.; Che, X. Salvianolic acid A as a multifunctional agent ameliorates doxorubicin-induced nephropathy in rats. Sci. Rep. 2015, 5, 12273. [CrossRef] [PubMed]

7. Hurle, M.R.; Yang, L.; Xie, Q.; Rajpal, D.K.; Sanseau, P.; Agarwal, P. Computational drug repositioning: From data to therapeutics. Clin. Pharmacol. Ther. 2013, 93, 335-341. [CrossRef] [PubMed]

8. Liu, Z.; Fang, H.; Reagan, K.; Xu, X.; Mendrick, D.L.; Slikker, W., Jr.; Tong, W. In silico drug repositioning: What we need to know. Drug Discov. Today 2013, 18, 110-115. [CrossRef] [PubMed]

9. Wang, L.; Xie, X.Q. Computational target fishing: What should chemogenomics researchers expect for the future of in silico drug design and discovery? Future Med. Chem. 2014, 6, 247-249. [CrossRef] [PubMed]

10. Ye, X.Y.; Ling, Q.Z.; Chen, S.J. Identification of a potential target of capsaicin by computational target fishing. Evid. Based Complement. Altern. Med. 2015, 2015, 983951. [CrossRef] [PubMed] 
11. Chen, S.J. A potential target of tanshinone iia for acute promyelocytic leukemia revealed by inverse docking and drug repurposing. Asian Pac. J. Cancer Prev. 2014, 15, 4301-4305. [CrossRef] [PubMed]

12. Ru, J.; Li, P.; Wang, J.; Zhou, W.; Li, B.; Huang, C.; Guo, Z.; Tao, W.; Yang, Y.; Xu, X.; et al. Tcmsp: A database of systems pharmacology for drug discovery from herbal medicines. J. Cheminform. 2014, 6, 13. [CrossRef] [PubMed]

13. Van de Waterbeemd, H.; Gifford, E. Admet in silico modelling: Towards prediction paradise? Nat. Rev. Drug Discov. 2003, 2, 192-204. [CrossRef] [PubMed]

14. Lipinski, C.A.; Lombardo, F.; Dominy, B.W.; Feeney, P.J. Experimental and computational approaches to estimate solubility and permeability in drug discovery and development settings. Adv. Drug Deliv. Rev. 2001, 46, 3-26. [CrossRef]

15. Leeson, P.D. Molecular inflation, attrition and the rule of five. Adv. Drug Deliv. Rev. 2016, 101, $22-33$. [CrossRef] [PubMed]

16. Tian, S.; Wang, J.; Li, Y.; Li, D.; Xu, L.; Hou, T. The application of in silico drug-likeness predictions in pharmaceutical research. Adv. Drug Deliv. Rev. 2015, 86, 2-10. [CrossRef] [PubMed]

17. Pei, T.; Zheng, C.; Huang, C.; Chen, X.; Guo, Z.; Fu, Y.; Liu, J.; Wang, Y. Systematic understanding the mechanisms of vitiligo pathogenesis and its treatment by qubaibabuqi formula. J. Ethnopharmacol. 2016, 190, 272-287. [CrossRef] [PubMed]

18. Zhang, W.; Tao, Q.; Guo, Z.; Fu, Y.; Chen, X.; Shar, P.A.; Shahen, M.; Zhu, J.; Xue, J.; Bai, Y.; et al. Systems pharmacology dissection of the integrated treatment for cardiovascular and gastrointestinal disorders by traditional Chinese medicine. Sci. Rep. 2016, 6, 32400. [CrossRef] [PubMed]

19. Hopkins, A.L. Network pharmacology: The next paradigm in drug discovery. Nat. Chem. Biol. 2008, 4, 682-690. [CrossRef] [PubMed]

20. Wang, J.; Gao, L.; Lee, Y.M.; Kalesh, K.A.; Ong, Y.S.; Lim, J.; Jee, J.E.; Sun, H.; Lee, S.S.; Hua, Z.C.; et al. Target identification of natural and traditional medicines with quantitative chemical proteomics approaches. Pharmacol. Ther. 2016, 162, 10-22. [CrossRef] [PubMed]

21. Cereto-Massague, A.; Ojeda, M.J.; Valls, C.; Mulero, M.; Pujadas, G.; Garcia-Vallve, S. Tools for in silico target fishing. Methods 2015, 71, 98-103. [CrossRef] [PubMed]

22. Tammali, R.; Srivastava, S.K.; Ramana, K.V. Targeting aldose reductase for the treatment of cancer. Curr. Cancer Drug Targets 2011, 11, 560-571. [CrossRef] [PubMed]

23. Ramana, K.V. Aldose reductase: New insights for an old enzyme. Biomol. Concepts 2011, 2, $103-114$. [CrossRef] [PubMed]

24. Du, G.H.; Qiu, Y.; Tian, Y.E.; Zhang, J.T. Prevention of galactose-induced cataractogenesis in rats by salvianolic acid A. Yao Xue Xue Bao 1995, 30, 561-566. [PubMed]

25. Hobbs, G.A.; Der, C.J.; Rossman, K.L. Ras isoforms and mutations in cancer at a glance. J. Cell Sci. 2016, 129, 1287-1292. [CrossRef] [PubMed]

26. Li, H.Y.; Li, Y.; Yan, C.H.; Li, L.N.; Chen, X.G. Inhibition of tumor growth by s-3-1, a synthetic intermediate of salvianolic acid A. J. Asian Nat. Prod. Res. 2002, 4, 271-280. [CrossRef] [PubMed]

27. McIlwain, C.C.; Townsend, D.M.; Tew, K.D. Glutathione s-transferase polymorphisms: Cancer incidence and therapy. Oncogene 2006, 25, 1639-1648. [CrossRef] [PubMed]

28. Qi, H.P.; Wei, S.Q.; Zhang, L.Q.; Gao, X.C.; Yu, N.N.; Bi, S.; Cui, H. Preventive effect of danshensu on selenite-induced cataractogenesis in cultured rat lens. Clin. Experiment. Ophthalmol. 2013, 41, 172-179. [CrossRef] [PubMed]

29. Gao, L.N.; Yan, K.; Cui, Y.L.; Fan, G.W.; Wang, Y.F. Protective effect of salvia miltiorrhiza and carthamus tinctorius extract against lipopolysaccharide-induced liver injury. World J. Gastroenterol. 2015, 21, 9079-9092. [CrossRef] [PubMed]

30. Lee, J.S.; Kim, H.G.; Han, J.M.; Kim, D.W.; Yi, M.H.; Son, S.W.; Kim, Y.A.; Choi, M.K.; Son, C.G. Ethanol extract of astragali radix and salviae miltiorrhizae radix, myelophil, exerts anti-amnesic effect in a mouse model of scopolamine-induced memory deficits. J. Ethnopharmacol. 2014, 153, 782-792. [CrossRef] [PubMed]

31. Zhang, J.; Lu, W.; Lei, Q.; Tao, X.; You, H.; Xie, P. Salvianolate increases heat shock protein expression in a cerebral ischemia-reperfusion injury model. Neural. Regen. Res. 2013, 8, 2327-2335. [PubMed]

32. Li, Q.; Li, X.; Li, C.; Chen, L.; Song, J.; Tang, Y.; Xu, X. A network-based multi-target computational estimation scheme for anticoagulant activities of compounds. PLoS ONE 2011, 6, e14774. [CrossRef] [PubMed] 
33. Hong, M.; Li, S.; Wang, N.; Tan, H.Y.; Cheung, F.; Feng, Y. A biomedical investigation of the hepatoprotective effect of radix salviae miltiorrhizae and network pharmacology-based prediction of the active compounds and molecular targets. Int. J. Mol. Sci. 2017, 18, 620. [CrossRef] [PubMed]

34. Cai, J.; Chen, S.; Zhang, W.; Zheng, X.; Hu, S.; Pang, C.; Lu, J.; Xing, J.; Dong, Y. Salvianolic acid A reverses paclitaxel resistance in human breast cancer mcf-7 cells via targeting the expression of transgelin 2 and attenuating pi3 k/akt pathway. Phytomedicine 2014, 21, 1725-1732. [CrossRef] [PubMed]

35. Zheng, X.; Chen, S.; Yang, Q.; Cai, J.; Zhang, W.; You, H.; Xing, J.; Dong, Y. Salvianolic acid A reverses the paclitaxel resistance and inhibits the migration and invasion abilities of human breast cancer cells by inactivating transgelin 2. Cancer Biol. Ther. 2015, 16, 1407-1414. [CrossRef] [PubMed]

36. Wang, X.J.; Xu, J.X. Salvianic acid A protects human neuroblastoma sh-sy5y cells against MPP+-induced cytotoxicity. Neurosci. Res. 2005, 51, 129-138. [CrossRef] [PubMed]

37. Bi, L.; Chen, J.; Yuan, X.; Jiang, Z.; Chen, W. Salvianolic acid A positively regulates pten protein level and inhibits growth of a549 lung cancer cells. Biomed. Rep. 2013, 1, 213-217. [PubMed]

38. Liu, X.; Ouyang, S.; Yu, B.; Liu, Y.; Huang, K.; Gong, J.; Zheng, S.; Li, Z.; Li, H.; Jiang, H. Pharmmapper server: A web server for potential drug target identification using pharmacophore mapping approach. Nucleic Acids Res. 2010, 38, W609-W614. [CrossRef] [PubMed]

39. Luo, H.; Chen, J.; Shi, L.; Mikailov, M.; Zhu, H.; Wang, K.; He, L.; Yang, L. Drar-cpi: A server for identifying drug repositioning potential and adverse drug reactions via the chemical-protein interactome. Nucleic Acids Res. 2011, 39, W492-W498. [CrossRef] [PubMed]

40. Warde-Farley, D.; Donaldson, S.L.; Comes, O.; Zuberi, K.; Badrawi, R.; Chao, P.; Franz, M.; Grouios, C.; Kazi, F.; Lopes, C.T.; et al. The genemania prediction server: Biological network integration for gene prioritization and predicting gene function. Nucleic Acids Res. 2010, 38, W214-W220. [CrossRef] [PubMed]

Sample Availability: Sample Availability: Not available.

(C) 2017 by the authors. Licensee MDPI, Basel, Switzerland. This article is an open access article distributed under the terms and conditions of the Creative Commons Attribution (CC BY) license (http:/ / creativecommons.org/licenses/by/4.0/). 